\title{
ФОРМАЛЬНЫЙ ПОДХОД К ОПТИМИЗАЦИИ РАЗМЕЩЕНИЯ ДИНАМИЧЕСКИХ МАССИВОВ ДАННЫХ МЕТОДОМ ПРЕДВАРИТЕЛЬНОГО РЕЗЕРВИРОВАНИЯ БЛОКОВ СТАТИЧЕСКОЙ ПАМЯТИ ЭВМ
}

\section{A FORMAL APPROACH TO OPTIMIZING THE PLACEMENT OF DYNAMIC DATA ARRAYS BY PRE-RESERVING BLOCKS OF STATIC COMPUTER MEMORY}

M. Tomaev

A. Gamidi

Summary. The problem of optimizing the placement of user data in multi-level computer memory by preemptive caching is Formalized. Two equivalent approaches to the formulation are given, and the relationship between them is demonstrated. The research is a theoretical basis for creating technologies and tools for creating optimal software products.

Keywords: optimization, program, model, search, quality, caching, static, dynamic.

\section{Ввемение}

$\Pi$ роблема эффективного размещения данных, используемых программой, в многоуровневой оперативной памяти ЭВМ (статическая память, стек, динамическая память) особенно актуальна для высоконагруженных вычислительных систем. С развитием языков программирования, включением в их состав библиотек, автоматизирующих наиболее распространенные операции по размещению и обработке данных, сформировалась тенденция размещения большей части пользовательских данных в динамической памяти ЭВМ. Однако, при выборе стратегии размещения данных в динамически выделяемых блоках ОП имеет необходимо учитывать возможные проблемы с производительностью. При высокой загрузке ОП возрастает сложность поиска эффективных вариантов дефрагментации, что приводит к снижению скорости обработки запросов на выделение новых блоков менеджером динамической памяти и, соответственно, томаев Мурат Хасанбекович

Доцент, Северо-Кавказский государственный горно-металлургический институт (государственный технологический университет), Владикавказ

Гамиди Артур Олегович

Аспирант, Северо-Кавказский государственный горно-металлургический институт (государственный технологический университет), Владикавказ a.gamidi@yandex.ru

Аннотация. Формализуется задача оптимизации размещения пользовательских данных в многоуровневой памяти ЭВМ методом упреждающего кэширования. Приведены два эквивалентных подхода к формулировке, демонстрируется взаимосвязь между ними. Исследование является теоретической основой для создания технологии и средств создания оптимальных программных продуктов.

Ключевые слова: оптимизация, программа, модель, поиск, качество, кэширование, статическая, динамическая.

становится причиной падения производительности операционной системы и прикладных программных пакетов. Таким образом одним из способов повышения производительности сложных программных комплексов является поиск эффективных стратегий перемещения данных из динамической «кучи» (heap) в сегменты фиксированного размера: сегмент глобальных данных либо стек.

В следующих листингах 1 и 2 (используется язык C++) приводится пример упреждающего кэширования, позволяющего избежать обращения к менеджеру динамической памяти до тех пор, пока размер массива не достигнет 1000 элементов.

Листинг 1. Исходная версия.

double *x = new [UserSize]; // выделение блока

....

delete [] x; //освобождение 
Листинг 2. Оптимизированная версия, использующяая упреждающее кэширование.

double ${ }^{*} x$;

static double $x$ Cache[1000];

$x=($ UserSize $>1000$ ? new [UserSize]: $x$ Cache);

...

If (x! = xCache) delete [] x; // освободение

В работе предлагается формализация метода упреждающего резервирования блоков динамической памяти. Демонстрируется оптимизационный характер задачи. Цель исследования - подготовка формальной базы для создания алгоритмов и инструментальных средств, автоматизирующих процессы поиска эффективных стратегий модификации исходного кода, позволяющих улучшить производительность проектируемых программных систем.

\section{Формулировка \\ оптимизационной заАачи}

\section{Обозначения.}

М - число динамических массивов данных;

V - верхняя граница доступного объема ОП;

$W_{i}$ - средний размер і-го динамического блока данHых;

$U_{i}$ - средний размер вспомогательного статического массива используемого для упреждающего кэширования і-го динамического массива;

$N_{i}$ - среднее количество операций выделения і-го динамического блока данных.

\section{Математическая модель}

В данной главе формулируется непрерывная оптимизационная задача (1), минимизирующая суммарное время выделения блоков динамической памяти, среднестатистический размер которых известен $\left(W_{i}\right)$. Демонстрируется переход к эквивалентной задаче (5), максимизирующей суммарное время размещения данных в статическом сегменте данных.

Сформулируем оптимизационную задачу, снижающую суммарное время выделения динамических блоков, используемых для размещения массивов данных.

$$
\left\{\begin{array}{l}
F=\sum_{i=1}^{m} N_{i} \frac{W_{i}-U_{i}}{s} \rightarrow \min ; \\
\sum_{i=1}^{m} Z_{i} W_{i} \leq V ; \\
\forall_{i}: 0 \leq U_{i} \leq W_{i} ; \\
z_{i}=0,1 ;
\end{array}\right.
$$

Преобразуем выражение в целевой функции так, чтобы левая сумма содержала только постоянные коэффициенты, а правая включала неизвестную $z_{i}$ :

$$
F=\sum_{i=1}^{m} N_{i} \frac{W_{i}}{s}-\sum_{i=1}^{m} N_{i} \frac{z_{i} U_{i}}{s} \rightarrow \min ;
$$

Уберем левую сумму, т.к. она представляет собой постоянную, не влияющую на оптимум:

$$
F=-\sum_{i=1}^{m} N_{i} \frac{z_{i} U_{i}}{s} \rightarrow \min ;
$$

Изменим знак перед суммой, и, соответственно, тип целевой функции с min на max:

$$
F=\sum_{i=1}^{m} N_{i} \frac{z_{i} U_{i}}{s} \rightarrow \max
$$

Упростим выражение, убрав постоянный коэффициент $1 / s$ и окончательно сформулируем оптимизационную модель в виде:

$$
\left\{\begin{array}{l}
F=\sum_{i=1}^{m} N_{i} z_{i} U_{i} \rightarrow \max ; \\
\sum_{i=1}^{m} z_{i} W_{i} \leq V ; \\
\forall_{i}: 0 \leq U_{i} \leq W_{i} ; \\
Z_{i}=0,1 ;
\end{array}\right.
$$

Смысл целевой функции в модели (5) заключается в максимизации суммарного объема данных, размещенного в статических массивах упреждающего кэширования вместо динамической (более медленной) памяти операционной системы.

\section{Зак^ючение}

Предложенный в работе подход к размещению данных позволит снизить частоту обращений и, соответственно, снизить вероятность неконтролируемого падения производительности сложных высоконагруженных систем. Предложенный формализм будет востребован при создании программных средств разработки оптимальных программных продуктов.

\section{ЛИТЕРАТУРЫ}

1. Побегайло А. П. Системное программирование в Windows. БХВ-Петербург, Спб.:2017

2. Уильямс Энтони. Параллельное программирование на С++ в действии. Практика разработки многопоточных программ. ДМК-Пресс, М:2012.

3. Томаев М.Х., Панарин В. Е. Выбор оптимального класса памяти для данных, используемых в программах на языке «С++». Материалы XIV международной научно-технической конференции «ИТ-технологии: развитие и приложения. г. Владикавказ, 2013», с. 215. 
4. Томаев М. Х. Использование оптимизационных моделей «экстремального программирования» в проектировании ПО. Выбор оптимальной стратегии макрозамен.ІТ-технологии: теория и практика. Материалы семинара. Владикавказ, 2017, стр. 39-55.

5. Томаев М.Х., Гамиди А. О. Формализация метода кэширования функций произвольного числа переменных. Наука и бизнес: пути развития. Научно-практический журнал. № 11(101), 2019. Стр. 75-79.

() Томаев Мурат Хасанбекович, Гамиди Артур Олегович (a.gamidi@yandex.ru ).

Журнал «Современная наука: актуальные проблемы теории и практики»

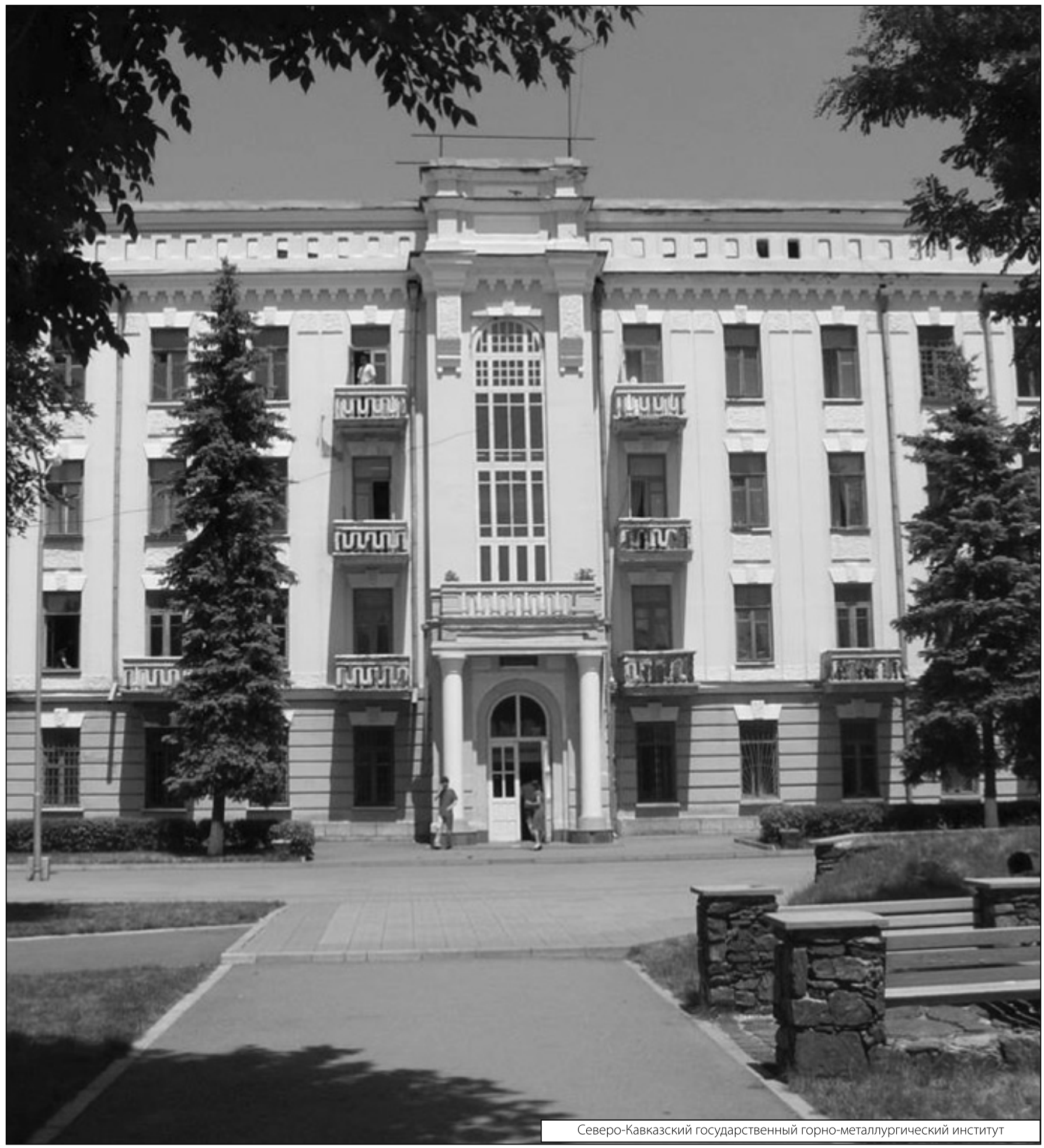

